\title{
A Human Resource Management Based Approach to Mitigating Traumatic Stress among Hospital Employees
}

\author{
Mark Brown \\ Department of Management and Leadership, Foster College of Business Administration, Bradley University, USA
}

Copyright $\bigcirc 2017$ by authors, all rights reserved. Authors agree that this article remains permanently open access under the terms of the Creative Commons Attribution License 4.0 International License

\begin{abstract}
This manuscript investigates hospitals' use of human resource management practices to mitigate traumatic stress among hospital employees. Initially, the concept of traumatic stress is described and various groups of hospital employees and their sensitivity to traumatic stress are discussed. The three groups of employees considered are hospital's clinical first responders, hospital's non-first responder clinical care personnel, and a third group composed of all remaining hospital employees. Lastly, the manuscript considers specific human resource interventions to address traumatic stress along with directions for future research.
\end{abstract}

Keywords Hospitals, Stress, Trauma, Interventions

\section{Introduction}

Stress is a widely researched topic which has long been identified as an important consideration for human resource professionals who wish to make their organizations more effective and efficient. [1] Stress is an organizational factor that is amenable to various interventions which can either ameliorate its existence and/or lessen its impact. Among organizations, hospitals have been noted to be among the most stressful work environments. [2] Sources of stress in hospitals include the nature of the work (e.g., seriously ill individuals), resource limitations (e.g., low staffing levels), and organizational complexity (e.g. role ambiguity). [3] Effects of stress include work dissatisfaction, low organizational commitment as well as a host of physical and physiological outcomes. [4]

While there are many sources of stress in hospitals, one of the most significant is traumatic stress. [5] Traumatic stress is the direct or indirect experience of any sudden or catastrophic event outside the range of normal human experience. [6] Traumatic stress is an important area of stress because, while it can and does manifest itself in terms of an attitudinal response (i.e., job attitudes), traumatic stress seems to be particularly problematic in terms of its potential physical and psychological consequences. [3,7,8] These profound individual consequences distinguish traumatic stress from traditional sources of work stress (e.g., role ambiguity, etc.) which have a comparatively more attitudinal response. [4] Moreover, from a human resource management perspective, these physical and psychological effects are important as they represent a source of heightened organizational costs due to increased employee healthcare costs, decreased productivity, and increased employee turnover. These increased organizational costs, along with the direct personal consequences of traumatic stress, make traumatic stress in hospitals an important area of consideration. Thus, this manuscript will investigate traumatic stress among hospital employees.

The manuscript will initially consider the concept of traumatic stress. Next, the manuscript will consider the effect and consequences of traumatic stress among various types of hospital employees. Following this, the manuscript will consider specific interventions useful in confronting traumatic stress along with suggestions for future research. From a research perspective this manuscript will be useful in introducing new areas of consideration for stress research. From a practical perspective this manuscript will assist hospitals in designing effective institutional responses to traumatic stress.

\section{Traumatic Stress in Hospitals}

Examples of traumatic stress in hospitals include direct and indirect exposure to the medical and psychological consequences of severe illness, accidental death and injury, violent homicide or suicide, workplace violence, and mass casualty events. [5,7,9] Although some employees may exhibit resilience to traumatic events as a result of self-selection to certain occupations, other employees may develop resilience through training and occupational socialization. $[9,10]$ For these resilient employees singular events of traumatic stress may be unproblematic whereas repetitive exposure to traumatic events may have strong cumulative effects. [4] Conversely, other employees may 
have little occupational experience with traumatic events and find even singularly experiences of traumatic stress problematic. [4]

\subsection{Diagnosis and Symptoms of Traumatic Stress}

Although a widely discussed topic, traumatic stress is not a formally recognized medical diagnosis. Clinically, short term manifestations of traumatic stress are appropriately and often categorized as adjustment disorders. [11] Adjustment disorders are the inability to respond, or a maladaptive reaction to identifiable stressful life events. [11] Adjustment disorders are temporary disorders of varying severity that occur as a reaction to overwhelming stress. [11] According to the Diagnostic and Statistical Manual of Mental Disorders (i.e., DSM-IV) symptoms of traumatic stress typically characterized as adjustment disorders include the display of agitation, depression, etc. that are severe enough to effect an individual's work or social life. Importantly, when characterized as an adjustment disorder, symptoms typically begin within three months and should not last longer than six months.

Longer term and more serious consequences of traumatic stress are usually considered as a form of post-traumatic stress disorders. [6] Post traumatic stress disorder (PTSD) is a more severe consequence of exposure to traumatic stress. The Diagnostic and Statistical Manual of Mental Disorders (i.e., DSM -IV) groups symptoms of PTSD into three main categories that include, (1) various experiences of reliving the event that disturbs daily functioning, (2) various forms of avoidance of activities that remind the individual of the traumatic event and, (3) emotional or physical arousal as a consequence of the traumatic event. [6] PTSD is distinguished from an adjustment disorder based upon the severity and length of existence of the symptoms displayed in response to the traumatic event. [11]

\section{Types of Hospital Workers and Traumatic Stress}

Effective consideration of traumatic stress in hospitals requires an analysis of its impact on particular groups of employees. Characteristics useful in evaluating traumatic stress's effects on particular employee groups include the group's level and type of exposure to traumatic stress as well as the group's self-selection, training, and socialization to confront traumatic stress. Three groups of hospital employees may be proposed to exist that vary along these dimensions (i.e., the employee group's level and type of exposure to traumatic stress and the employee group's self-selection, training, and socialization to confront traumatic stress). These groups are hospital's clinical first responders, hospital's non-first responder clinical care personnel, and a third category composed of all remaining hospital employees.

\subsection{Hospital Clinical First Responders}

Hospital clinical first responders have the first employee encounter with patients. Examples of these employees include clinical staff in the emergency room along with clinical staff in other areas that have early admission interactions with seriously injured patients (i.e., radiology staff, surgery staff, etc.). Hospital's clinical first responders are frequently exposed to traumatic stress. These employees experience firsthand the consequences of violent and accidental injury and are among the most taxed employees during mass casualty events. [12]

Clinical first responders demonstrate a significant resistance to traumatic stress as they likely self-select themselves into occupations with high traumatic stress. [9] Clinical first responders understand their work environment as well as their potential exposure to traumatic stress and have made a decision to work in this environment. Clinical first responders have typically been socialized through training - and learned through experience - how to deal with traumatic stress. [9] Although clinical first responders demonstrate a high tolerance for traumatic stress, the cumulative effects of traumatic stress may be problematic. Thus, although these employees have the ability to deal with individual incidents of stress, some may experience dysfunctional consequences due to repetitive exposure to traumatic stress. [13] Differential responses are explained by individual employee's types and levels of social support as well as differences in coping and other abilities. [14]

\subsection{Hospital Non-first Responder Clinical Care Personnel}

This group of employees is composed of clinical personnel who do not have initial point of care contact with patients. Employees representative of this group include clinical personnel in patient treatment departments as well as those in counseling and social service areas. These employees do not experience firsthand the consequences of traumatic events but rather the secondary effects once initial care has been administered. Thus, whereas clinical first responder employees vividly experience a traumatically injured patient, non-first responder clinical care personnel experience them in a more sanitized form. While the patient may arrive at the hospital bleeding and dirty for the clinical first responder, non-first responder clinical care personnel receive patients in a comparatively less traumatic state more often times bandaged and clean.

Like clinical first responder employees these employees (i.e., non-first responder clinical care personnel) frequently have training and socialization to deal with traumatically stressful situations. Many of these employees have worked in clinical first responder positions and may have experienced traumatic stress. Interestingly, unlike many clinical first responders, some non-first responder clinical care personnel may have intentionally self-selected themselves into settings where they experience less 
traumatic stress. This potentially suggests some of these employees are more susceptible to the consequences of traumatic stress than clinical first responder employees. Fortunately, due to the nature of their work, non-first responder clinical care personnel are less frequently exposed to traumatic stress.

\subsection{Other Hospital Employees}

Beyond hospital's clinical first responders and non-first responder clinical care personnel is a third group composed of the hospital's remaining employees. Many of these employees perform jobs which are common across other types of organizations (e.g., accountants, computer programmers, cooks).[15] These employees often choose to work in a hospital based upon the quality of a job offer rather than a desire to pursue a health care career. These employees are differentiated from other employees (i.e., clinical first responders and non-first responder clinical care personnel) in that they are neither trained nor socialized to deal with traumatic stress. While these employees have had little exposure, training, or socialization to deal with traumatic stress - not all will experience difficulties when confronted with traumatic stress. Indeed, the same social support and individual difference characteristics that explain other employee's responses to traumatic stress also influence these employee's responses. [14] Nevertheless, potential negative consequences must be recognized among these employees due to their limited exposure, training, and socialization to traumatic stress.

While traumatic stress is less common among this group of employees, caution should be exercised when evaluating these employees potential for traumatic exposure. For instance, while an admitting clerk is considered part of this employee group, their working in the emergency room might cause them to experience traumatic stress levels typical of a clinical first responder. Moreover, due to these employees' physical proximity to a hospital's operational environment, it is likely they will eventually experience some type of traumatic stress. This is particularly the case in mass causality situations where operational exigencies may require non clinical personnel to perform unskilled clinical roles.

\section{Organizational Interventions to Deal with Traumatic Stress}

Identifying particular groups of employees and their sensitivity to traumatic stress is useful in devising interventions to address traumatic stress. In general, the most effective human resource management response to any organizational challenge is one that is as broad and encompassing as possible. Thus, the goal in developing a human resource response to traumatic stress should be to devise a general response with specific interventions to address the unique needs of specific groups of employees.

\subsection{Organizational Level Human Resource Practices to Address Traumatic Stress}

Initial employee orientation is the best opportunity for hospitals to adopt an organization wide approach to confronting traumatic stress. During orientation all employees should be made aware of traumatic stress. Training should include basic knowledge of traumatic stress, its recognition, and its potential consequences. [3,5,6,7] Specific employee groups (i.e., clinical first responders, non-first responder clinical care personnel, and other hospital employees) should be provided with traumatic stress information relevant to their group. Employees should be reminded that traumatic stress occurs in hospitals and that negative reactions to traumatic stress should never be viewed as a sign of weakness.

In terms of particular employee groups, clinical first responder employees should be made aware of the cumulative effects of traumatic stress whereas non-clinical first responders should be reminded of the potential negative effects of traumatic stress. Training for the third group of hospital employees (i.e., all other hospital employees) should focus on recognition of the symptoms of exposure to traumatic stress along with strategies to cope and deal with traumatically stressful events. Training for these employees should be designed to compensate for their lack of training and socialization in dealing with traumatic stress.

One promising area of addressing traumatic stress is the creation of online traumatic stress training programs. Online training programs offer a convenient and easily accessible way for hospitals to prepare employees for traumatic stress. Programs can be designed to provide initial as well as specific training for individuals who have recently experienced traumatic stress. A recent study investigating the use of online training to assist military veterans suffering from PTSD found that online training showed promise as a means of delivering effective early PTSD treatment. [16]

\subsection{Individual Level Human Resource Benefits and Interventions to Deal with Traumatically Stressful Events}

Several individual level therapeutic techniques may be helpful in confronting the adverse consequences of specific traumatic events. In particular, research suggests the usefulness of cognitive behavioral therapy [17] and eye movement desensitization and reprocessing therapy. [18] Each of these therapeutic techniques may be effective in addressing the needs of individuals who have experienced traumatic stress. [19]

Empirical support has consistently demonstrated the effectiveness of cognitive behavioral therapy (CBT) in treating anxiety disorders such as PTSD. [20] CBT assumes the way an individual makes sense of an event is of greater consequence than the actual experiencing of the event itself. [21] Based on extensive experience treating military veterans suffering from PTSD, the United States Department of 
Veterans Affairs [22] recommends cognitive therapy and exposure therapy as two forms of CBT effective in treating PTSD. According to the United States Department of Veteran Affairs [22], cognitive therapy alleviates the stress and symptoms of PTSD by helping sufferers understand and change how they think about their traumatic exposure. Relatedly, exposure therapy seeks to lessen the effect of stressful fears associated with memories of traumatic events. Specifically, through exposure to fearful memories, PTSD suffers learn to alleviate stress and symptoms associated with thoughts, feelings, and situations reminiscent of traumatic events.

Eye movement desensitization and reprocessing (EMDR) therapy is based on the concept that negative thoughts, feelings, and behavior associated with a traumatic event are a consequence of memories of the event that have not been properly processed due to the traumatic nature of the event. [23] The therapy focuses upon having the individual simultaneously focus upon aspects of the traumatic event while also focusing upon a stimulus which is most commonly implemented to generate bilateral movements of the eye. [24] Although the operative mechanism of the EMDR remains unclear, it is believed that concurrent attention to both the traumatic event and the unrelated stimulus serves to lessen the effect of the memory of the traumatic event. [25] Specifically, it is believed that the simultaneous attention to the unrelated stimuli lessens the vividness of the memory of the traumatic event such that, while the PTSD sufferer still remembers the traumatic event, it is subsequently recalled in a less upsetting manner. [23]

Although, hospitals may address traumatic stress by providing employees access to specific therapeutic techniques, they must still assure employees fundamentally have access to competent mental health professionals with knowledge of a range of clinical interventions. In the end, no one specific clinical technique is universally appropriate for treating exposure to traumatic stress. [26] Rather, it is access and, where necessary, treatment by trained mental health professionals that assures the most positive outcomes.

\section{Conclusions}

This manuscript has evaluated hospitals' use of human resource management practices to address traumatic stress among hospital employees. Employees' career self-selection along with their socialization and training to deal with traumatic stress likely explain the significance and incidence of traumatic stress among various groups of hospital employees. Hospital orientation programs which focus on the traumatic stress needs of all employees, while also addressing the needs of specific groups of employees, are likely one human resource management practice useful in addressing traumatic stress. Similarly, when traumatically stressful events occur, and employees are adversely affected, hospitals must provide human resource benefits such as access to trained mental health professionals.
Future research should empirically consider the effectiveness of hospital orientation programs that provide employees with information about traumatic stress. When hospitals provide this material it would be useful to understand the differential benefits of information relevant to specific employee groups versus general information applicable to all employees. Similarly, future research should also investigate the usefulness of various clinical interventions in assisting specific groups of hospital employees in confronting traumatic stress. Specifically, it would be useful to understand if some interventions are more appropriate for certain employee groups (i.e., clinical first responders, non-first responder clinical care personnel, and other hospital employees) depending upon their career self-selection, and/or training and socialization to deal with traumatic stress.

\section{REFERENCES}

[1] Avey, J.B., Luthans, F, \& Jenson, S.M. (2009). Psychological capital: a positive resource for combating employee stress and turnover. Human Resource Management, 48(5), 677-693.

[2] Buttigieg, S.C., West, M.A., \& Dawson, J.F. (2011). Well structured teams and the buffering of hospital employees from stress. Health Services Management Research, 24, 203-212.

[3] Mealer, M., Burnham, E., Goode, C., Rothbaum, B. \& Moss, M. (2009). The prevalence and impact of post traumatic stress disorder and burnout syndrome in nurses. Depression and Anxiety, 26(12), 118-1126.

[4] Adriaenssens, J., De Gucht, V. \& Maes, S. (2014). Determinants and prevalence of burnout among nurses: A Systematic review of 25 years of research. International Journal of Nursing Studies, (http://dx.doi.org/10.1016/j.jnurs tu.2014.11.004).

[5] Mealer, M., \& Jones, J. (2013). Posttraumatic stress disorder in the nursing population: A concept analysis. Nursing Forum, 48(4), 279-288.

[6] National Institute of Mental Health (2012). Post-traumatic stress disorder. Retrieved from http://www.nimh.nih.gov/health/publications/post-traumaticstress-disorder-ptsd/complete-index.shtml.

[7] Janda, R. \& Jandova, E. (2015). Symptoms of posttraumatic stress disorder, anxiety and depression among Czech critical care and general surgical and medical ward nurses. Journal of Research in Nursing, 20(4), 298-309.

[8] Laschinger, H.K.S. \& Nosko, A. (2015). Exposure to workplace bullying and post-traumatic stress disorder symptomology: The role of protective psychological resources. Journal of Nursing Management, 23(2), 252-262.

[9] North, C.S., Tivis, L., NcMillen, C., Pfefferbaum, B., Spitznagel, E.L., Cox, J., Nixon, S., Bunch, K.P. \& Smith, E.M. (2002). Psychiatric disorders in rescue workers after the oklahoma city bombing, American Journal of Psychiatry, 159(5), 857-859. 
[10] Mealer, M., Jones, J., Newman, J., McFann, K.K. \& Rothbaum, B. (2011). The presence of resilience is associated with a healthier psychological profile in intensive care unit (ICU) nurses: Results of a national survey. International Journal of Nursing Studies, 49, 292-299.

[11] Casey, P. \& Bailey, S. (2011). Adjustment disorders: the state of the art. World Psychiatry, 10(1), 11-18.

[12] Lavoie, S., Talbot, L.R. \& Mathieu, L. (2011). Post-traumatic stress disorder symptoms among emergency nurses: their perspective and a 'tailor made' solution. Journal of Advanced Nursing, 67(7), 1514-1522.

[13] Lombardo, B. \& Eyre, C. (2011). Compassion fatigue: a nurse's primer. OJIN: The Online Journal of Issues in Nursing, 16(1), Manuscript 3.

[14] Naomi, B. \& Glen, D.C. (1987). Posttraumatic stress disorder: the stressor criterion. The Journal of Nervous and Mental Disease, 175(5), 255-316.

[15] Shi, L., \& Singh, D. A. (2017). Essentials of the U.S. health care system (4th ed.). Burlington, MA: Jones \& Bartlett Learning.

[16] Engel, C.C., Litz, B., Magruder, K.M., Harper, E.H., Gore, K., Stein, N., Yeager, D., Liu, X. \& Coe, T.R. (2015). Delivery of self training and education for stressful situations (DESTRESS-PC): a randomized trial of nurse assisted online self-management for PTSD in primary care. General Hospital Psychiatry 37, 323-328.

[17] Kar, N. (2011) Cognitive behavioral therapy for the treatment of post-traumatic stress disorder. Neuropsychiatric Disease and Treatment (7) 167-181.

[18] Shapiro, F (1989). Efficacy of the eye movement desensitization procedure in the treatment of traumatic memories. Journal of Traumatic Stress 2(2): 199-223.

[19] Bisson, J.I., Ehlers, A. Matthews, R., Pilling, S., Richards, D. \& Turner, S. (2007). Psychological treatments for chronic post-traumatic stress disorder. The British Journal of Psychiatry, 190(2), 97-404.

[20] McMain, S., Newman, M.G., Segal, Z.V. \& DuRubies, R.J. (2015). Cognitive behavioral therapy: Current status and future research directions. Psychotherapy Research 25(3), 321-329.

[21] Mandler, H., \& Kingdon, D. (2015). The evolution of cognitive behavioral-therapy for psychosis. Psychological Research and Behavior Management, 8, 63-69.

[22] United States Department of Veteran Affairs (n.d.). Treatment of PTSD. Retrieved from http://www.ptsd.va.gov/public/treatment/therapy-med/treatm ent-ptsd.asp

[23] EMDR Institute (n.d.). What is EMDR. Retrieved from http://www.emdr.com/what-is-emdr.

[24] World Health Organization (2013). Guidelines for the management of conditions specifically related to stress. Retrieved from https://www.ncbi.nlm.nih.gov/books/NBK159725/

[25] Arkowitz, A. \& Lillenfield, S.O. (2007). EMDR: Taking a closer look. Scientific American. Retrieved from http://www.scientificamerican.com/article/emdr-taking-a-clo ser-look.

[26] Kagee, A. (2002). Concerns about the effectiveness of critical incident stress debriefing in ameliorating stress reactions. Critical Care, 6(1), 88. 